\title{
BMJ Open Is postoperative bracing after pedicle screw fixation of spine fractures necessary? Study protocol of the ORNOT study: a randomised controlled multicentre trial
}

\author{
Arjen Johannes Smits, ${ }^{1}$ Jaap Deunk, ${ }^{1}$ Agnita Stadhouder, ${ }^{2}$ Mark Cornelis Altena, ${ }^{3}$ \\ Diederik Hendrik Ruth Kempen, ${ }^{3}$ Frank Willem Bloemers ${ }^{1}$
}

To cite: Smits AJ, Deunk J, Stadhouder A, et al. Is postoperative bracing after pedicle screw fixation of spine fractures necessary? Study protocol of the ORNOT study: a randomised controlled multicentre trial. BMJ Open 2018;8:e019596. doi:10.1136/ bmjopen-2017-019596

\section{- Prepublication history for} this paper is available online. To view these files, please visit the journal online (http://dx.doi. org/10.1136/bmjopen-2017019596).

Received 13 September 2017 Revised 10 December 2017 Accepted 14 December 2017

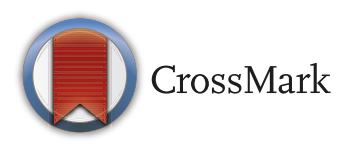

${ }^{1}$ Department of Trauma Surgery, VU University Medical Center, Amsterdam, The Netherlands ${ }^{2}$ Department of Orthopedic Surgery, VU University Medical Center, Amsterdam, The Netherlands

${ }^{3}$ Department of Orthopedic Surgery, Onze Lieve Vrouwe Gasthuis, Amsterdam, The Netherlands

Correspondence to Dr. Arjen Johannes Smits; aj.smits@vumc.nl

\section{ABSTRACT}

Introduction The most common surgical treatment of traumatic spine fractures is through a posterior approach using pedicle screws and rods. Postoperative treatment protocols including the use of postoperative orthoses however differ between hospitals and surgeons. A threepoint hyperextension orthosis is designed to support proper posture and unload the anterior column. Some motion remains when wearing an orthosis, and its main value in postoperative treatment is therefore believed to be pain relief and patient confidence. This could consequently shorten recovery time. On the other hand, an orthosis could also lead to muscle weakness and slow down recovery. Any orthosis-related complications might also be avoided. Additionally, recent studies on conservative fracture treatment show no difference in radiological outcomes with or without an orthosis. To date, no randomised studies have been performed on the use of postoperative orthoses.

Methods and analysis Patients undergoing posterior fixation with pedicle screws for a traumatic thoracolumbar fracture (T7-L4) will be included in this randomised controlled multicentre non-inferiority trial. Forty-six patients will be randomised 1:1 to one of the two parallel groups; one group will wear a postoperative orthosis for 6 weeks followed by 6 weeks of weaning and one group will not wear an orthosis. The primary outcome is pain at 6 weeks reported on the Numerical Rating Scale. Secondary outcomes consist of pain on other moments, analgesic use, complications and length of hospital stay, quality of life (EuroQuol 5 Dimensions), back pain-related function (Oswestry Disability Index) and radiological outcomes with a follow-up of 1 year. Orthosis compliance is monitored weekly in the orthosis group. Ethics and dissemination The institutional review board (METc VUmc) approved this study on 11 October 2016 under case number 2016.389. After completion of the trial, the results will be offered to an international scientific journal for peer-reviewed publication.

Trial registration number NCT03097081 and NTR6285; Pre-results.

\section{BACKGROUND}

A lot of research is currently done involving the best operative and non-operative
Strengths and limitations of this study

- This is the first randomised study to make an evidence-based recommendation on the use of a postoperative orthosis after posteriorly fixated traumatic spine fractures.

- The results might lead to a change in protocols and prevent unnecessary patient inconvenience and costs.

- Patients randomised to an orthosis might not use it as often as prescribed, therefore weekly monitoring of orthosis compliance is introduced in this study.

- Pain measurement (primary outcome) is subject to momentary peaks and dips. To obtain reliable average values and rule out distorted maximum or minimum values, pain is reported three times daily for four consecutive days.

treatment of traumatic thoracolumbar spine fractures. Both surgical stabilisation and the use of orthoses play an important role in the treatment of thoracolumbar fractures. The most common surgical intervention is posterior fixation with pedicle screws and rods. Guidelines on postoperative care remain ambiguous, and the role of an orthosis differs depending on hospital and surgeon's preference. ${ }^{1-3}$ An orthosis is designed to support proper posture and unload the anterior vertebral column through a three-point fixation. ${ }^{4}$ It does, however, not provide rigid stability or complete immobilisation of the spine.

While operatively treated fractures gain intrinsic stability from the implanted fixation material, an orthosis is still commonly used in postoperative care by many surgeons. ${ }^{3}{ }^{5-9}$ The additional value is supposed to be pain relief, patient confidence and reducing the load on implanted hardware. An orthosis is not likely to influence hospital stay duration 
as shorter hospital stay was not observed in one study ${ }^{10}$ and even longer hospital stay was reported in another. ${ }^{11}$ Other disadvantages are possible orthosis-related complications such as muscle atrophy, stiffness, skin irritation, thromboembolism and impaired respiration. ${ }^{10-12}$

Studies that reported not to use an orthosis postoperatively are scarce, ${ }^{312} 13$ while the benefits seem obvious. One study encouraged immediate mobilisation without orthosis to allow a more rapid return to activities and avoid complications. ${ }^{12}$ Furthermore the authors found that loss of kyphosis correction did not differ from studies that used bracing after surgery. No weaning from the brace could lead to quicker return to daily activities and work which might lead to faster improvement of quality of life (QOL) and back pain-related function while reducing costs for society. Finally, (rare) orthosis-related complications are avoided.

The use of an orthosis has been studied postoperatively after lumbar spine surgery for degenerative conditions ${ }^{1415}$ and for conservative treatment of thoracolumbar fractures. ${ }^{11} 1617$ In one study on lumbar surgery for degenerative conditions, it was found to promote arthrodesis. ${ }^{14}$ No differences were shown concerning pain, adverse events, functional outcomes, radiographic consolidation and kyphotic progression. One study on conservative treatment reported improved pain and functional scores when using a brace compared with physical therapy. ${ }^{18} \mathrm{~A}$ recent systematic review on postoperative bracing showed comparable results for both groups except slightly more kyphosis correction loss and less pseudoarthrosis in the group with postoperative bracing. ${ }^{3}$ It has to be noted that this review is probably not reliable to translate into clinical practice as it contains large heterogeneity, combining all types of studies and (operative) treatments.

Possibly due to the fact that there are no clinical studies to date to prove or disprove the additional value after surgical stabilisation of thoracolumbar fractures, a postoperative orthosis is still often common practice. The aim of this randomised trial is to make the first evidence-based recommendation on postoperative use of an orthosis for posteriorly stabilised traumatic thoracolumbar fractures. We hypothesise that postoperative pain without an orthosis will not be worse compared with postoperative pain with an orthosis. Additionally, it is hypothesised that there will be no influence on fracture collapse, kyphosis correction, complications and functional outcomes as compared with postoperative treatment without an orthosis.

\section{METHODS AND ANALYSIS \\ Study design}

The study is set up as a parallel 1:1 group randomised controlled multicentre non-inferiority trial. It will take place in the Netherlands in one initiating academic hospital and one participating regional centre; VU University medical centre Amsterdam (Department of Trauma Surgery and Department of Orthopaedic Surgery) and Onze Lieve Vrouwe Gasthuis Amsterdam
(Department of Orthopaedic Surgery). The study protocol is written according to the Standard Protocol Items: Recommendations for Interventional Trials (SPIRIT) guidelines ${ }^{19}$ and the study will be reported according to the Consolidated Standards of Reporting Trials guidelines. ${ }^{20}$

\section{Recruitment}

Patients will be initially recruited in one of two participating centres by the treating surgeon once the decision for posterior fixation has been made. Study-specific and legal information about the study is given and permission to be contacted by one of the study team members is requested. Within 24 hours patients will be contacted again by their treating doctor or another trained study team member to discuss any further questions and, if applicable, sign informed consent.

\section{Eligibility criteria}

Patients between 18 and 65 years of age with a traumatic thoracolumbar (T7-L4) fracture that will undergo or have $<24$ hours ago undergone posterior fixation (short and long segments) using pedicle screws and rods are potentially eligible. Patients with a neurological injury or psychiatric comorbidity are excluded because it is very likely that this influences QOL outcomes. Further exclusion criteria are reported in table 1.

\section{Interventions}

Two groups are created; one group with a postoperative ORthosis versus a group with NO orThosis (ORNOT), figure 1. The study intervention is no postoperative orthosis, the orthosis group serves as control while this is the current postoperative protocol. The three-point hyperextension orthosis is used which supports vertebra T7 to L4. After the operation, patients have at least 6 hours of bed rest, after which they can try and mobilise as tolerated with the help of a physiotherapist. After this point, they are permitted to walk, if tolerated. According to the randomisation, this will be done with or without an orthosis. The group randomised to an orthosis is required to wear this when in vertical position (standing, walking and sitting up straight) for 6 weeks, followed by 6 weeks of gradual weaning. Weaning of the orthosis is as tolerated, based on pain and physical condition of the patient. Because potential differences exist between patients in the amount of perceived pain, confidence and range of motion, weaning is a process that is patient specific. The orthosis does not need to be worn when the patient is lying down. The orthosis comes in three standard sizes (small, medium and large) and will be adjusted manually to the patient by a specialised nurse. Both groups will receive standard postoperative protocol pain medication if needed and discharge will be based on clinical improvement. Every participant to the study has the right to withdraw from the study at any time without further argumentation. 
Table 1 Inclusion and exclusion criteria for the ORNOT trial

\begin{tabular}{ll}
\hline Inclusion & Exclusion \\
\hline 18-65years & Inadequate knowledge of the Dutch language to fill in questionnaires \\
Traumatic fracture T7-L4 & Complete or partial spinal cord injury (ASIA A-D) \\
Fracture type AO A2-C & (Additional) Anterior surgical stabilisation through thoracotomy or \\
Surgical posterior fixation with pedicle screws & lumbotomy \\
& Thoracolumbar fracture of non-traumatic aetiology (eg, pathological and \\
& infectious) \\
& Osteoporosis; using bisphosphonates or positive DEXA \\
& - ISS $\geq 16$ \\
& Brain injury AIS $\geq 4$ \\
& Not able to walk before the fracture \\
& Unable to come to the outpatient clinic \\
& Psychiatric comorbidity \\
& Inability to wear an orthosis, for example: \\
& - BMI $>35$ \\
& - Thoracoabdominal wounds \\
& - Pre-existing spine deformity which impairs the use of an orthosis \\
\hline
\end{tabular}

AIS, Abbreviated Injury Score; AO, AO Spine fracture classification; ASIA, American Spinal Injury Association score; BMI, body mass index; DEXA, Dual Energy X-ray Absorptiometry; ISS, Injury Severity Score; L, lumbar; ORNOT, ORthosis versus a group with NO orThosis; T, thoracic.

Patients in the orthosis group are monitored for adherence to their orthosis use. They are asked and reminded several times, to register their weekly orthosis compliance during 12 weeks on a week diary that is handed out at inclusion. Patients randomised to no orthosis cannot be monitored for their adherence.

\section{Primary outcome}

The primary outcome parameter is pain at 6 weeks, measured on the Numerical Rating Scale (NRS) and compared between the two groups. A clinically significant difference is defined as two points on the NRS scale. This difference of two points is based on previous literature $^{2122}$ on pain and based on meetings with the hospital's pain exerts. If an orthosis affects postoperative pain, we hypothesise this effect will be the largest at 6 weeks postoperatively. Surgical side effects such as wound pain will then mostly be disappeared and the fracture will not yet be fully consolidated which should adequately demonstrate the effect of an (or no) orthosis on back pain. Weaning from the orthosis will also be started at 6 weeks. The NRS score (with 0-10 range) has shown similar psychometric properties to the Visual Analogue Scale (VAS), ${ }^{21}{ }^{23-25}$ is preferred over the VAS score by patients $^{26}$ and has shown applicable to back pain. ${ }^{27-29}$ In order to obtain reliable average values and rule out distorted maximum or minimum values, patients are required to fill in the NRS score for four consecutive days, three times a day at each measurement moment (2, 6 and 12 weeks). It has been shown that a composite pain score demonstrates adequate reliability and excellent validity as a measure of average pain. ${ }^{30}$ The primary outcome, pain at 6 weeks, will consequently be the mean of 12 values per patient.

\section{Secondary outcomes}

Pain (NRS) on other measurement moments and analgesics

Pain measured on other moments than the primary moment will function as secondary outcomes (figure 2). The moment 'day of discharge' is defined as day 1 to (maximum) day 10. Groups will be compared at each moment and the total course of pain development over 12 weeks will be compared.

Analgesics are additionally registered at the pain measurement moments. Patients have to answer whether they at that moment use analgesics for back pain (yes/ no), and if yes, what medication (acetaminophen, non-steroidal anti-inflammatory drugs, opioids or a combination). The medication used will be compared between groups.

Quality of life

QOL will be measured (figure 2) using the EuroQuol 5 Dimensions (EQ-5D-5L) questionnaire which has shown validity for low back pain. ${ }^{31}$ The EQ-5D is a standardised measure of health status that consists of five domains: mobility, self-care, usual activities, pain/discomfort and anxiety/depression. Each domain can be scored on five levels: no problems, slight problems, moderate problems, severe problems and extreme problems/unable. Finally, the questionnaire consists of a VAS score which can be scored from 1 to 100 to be used as a quantitative measure of general health. An index value of overall health can be calculated for each patient using the five dimension scores and a country-specific value set, which is available for the Netherlands. Groups will be compared on domain, VAS and index scores. Furthermore, EQ-5D scores will be compared with scores of the Dutch general population. ${ }^{32}$ 


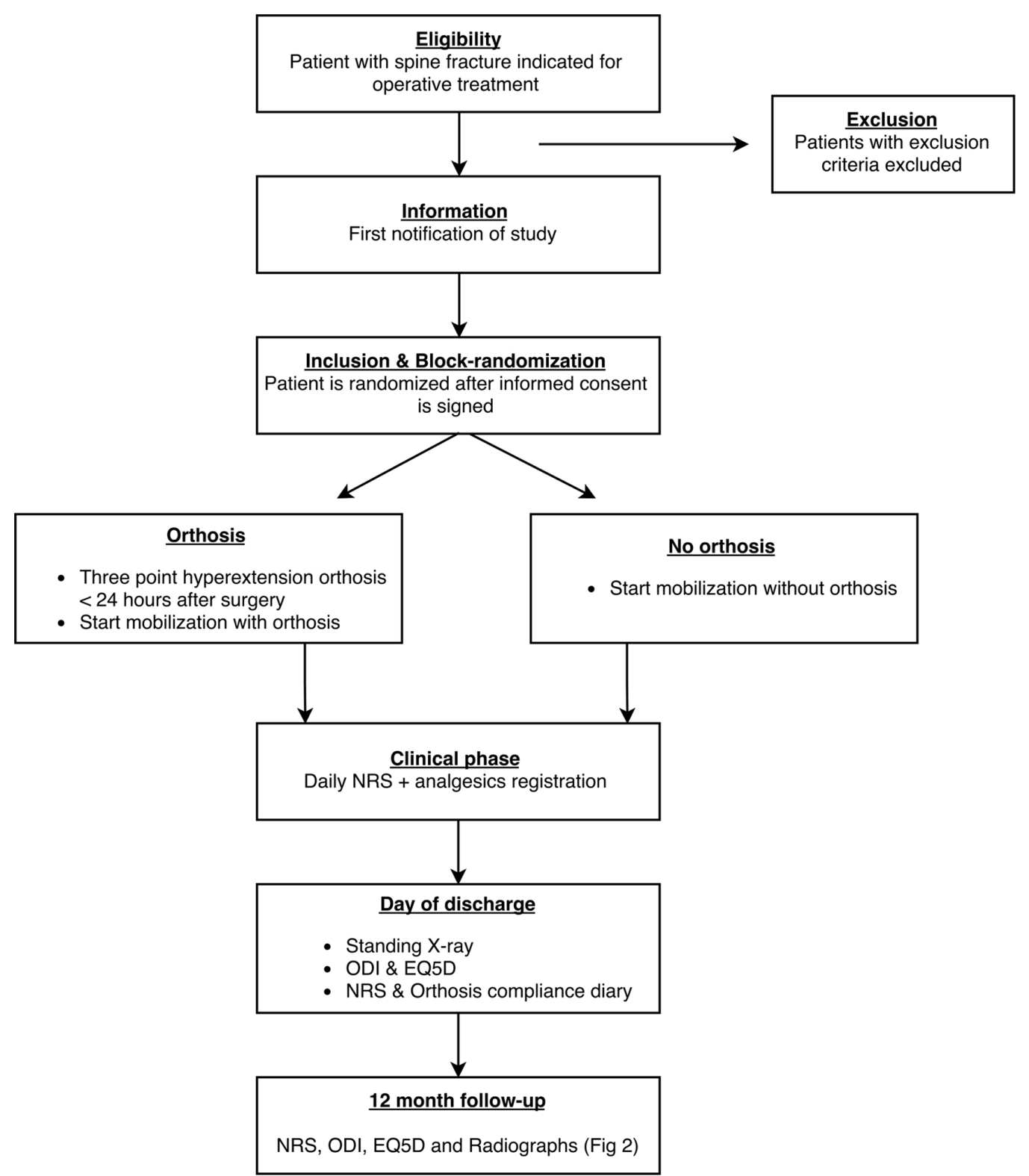

Figure 1 Flow chart for patients from presentation until final follow-up. EQ-5D, EuroQuol 5 Dimensions; NRS, Numerical Rating Scale; ODI, Oswestry Disability Index.

\section{Back pain-related function}

The Oswestry Disability Index (ODI) ${ }^{33}$ is validated for ${ }^{27}{ }^{34}$ and will be used to measure condition-specific back pain (figure 2). The 'day of discharge' value will be determined on day 1 to (maximum) day 10 . Originally designed for chronic low back pain but due to lack of specific scoring instruments, now it is also often used for evaluation of treated traumatic thoracolumbar fractures. ${ }^{35}$ The ODI questionnaire consists of 10 questions with each six answer options that are rated from 0 to 5 points from which a summary score is calculated.

Kyphosis and fracture consolidation

Kyphosis will be measured (figure 2) using the Cobb angle which has proven the best interobserver and intraobserver reliability ${ }^{36}$; defined as the angle between the upper endplate of the vertebra above and the lower endplate of the vertebra below the fractured vertebra. This will be done on CT scan (mid-sagittal slide) preoperatively and at follow-up moments on standing X-ray. Follow-up radiographs are made at the discretion of the treating surgeon and generally follow a standard pattern (figure 2). Fracture consolidation will be assessed at the same time of kyphosis assessment using the same radiographic images. Kyphosis and fracture consolidation measurements will be done at the end of follow-up by an outcome assessor (orthopaedic/trauma surgery resident) blinded to patient allocation. Additionally, if any implant failure is seen on follow-up radiographs, this will be recorded under complications. In difficult cases, an independent experienced spine surgeon will be consulted. 


\begin{tabular}{|c|c|c|c|c|c|c|c|c|}
\hline \multirow[b]{3}{*}{ TIMEPOINT } & \multirow{3}{*}{$\begin{array}{c}\text { Enrolment } \\
\begin{array}{l}\text { Clinical } \\
\text { phase }\end{array}\end{array}$} & \multicolumn{7}{|c|}{ STUDY PERIOD } \\
\hline & & \multirow{2}{*}{$\begin{array}{l}\text { Allocation } \\
<24 \mathrm{~h} \text { post } \\
\text { operative }\end{array}$} & \multirow[b]{2}{*}{$\begin{array}{l}\text { Day of } \\
\text { discharge } \\
\text { (day } 1 \text { to } \\
\text { max. 10) } \\
\end{array}$} & \multicolumn{5}{|c|}{ Post-allocation } \\
\hline & & & & $\begin{array}{c}2 \\
w k s\end{array}$ & $\begin{array}{c}6 \\
w k s\end{array}$ & $\begin{array}{c}12 \\
w k s\end{array}$ & $\begin{array}{c}6 \\
m t s\end{array}$ & $\begin{array}{c}12 \\
m t s\end{array}$ \\
\hline \multicolumn{9}{|l|}{ ENROLMENT: } \\
\hline \multirow{2}{*}{$\begin{array}{l}\text { Eligibility screen } \\
\text { Informed consent }\end{array}$} & $\mathrm{x}$ & & & & & & & \\
\hline & & $\mathrm{X}$ & & & & & & \\
\hline Allocation & & $X$ & & & & & & \\
\hline \multicolumn{9}{|l|}{ INTERVENTIONS: } \\
\hline \multicolumn{9}{|l|}{ Orthosis } \\
\hline \multicolumn{9}{|l|}{ No Orthosis } \\
\hline \multicolumn{9}{|l|}{ ASSESSMENTS: } \\
\hline \multirow{2}{*}{ Baseline variables } & $x$ & & & & & & & \\
\hline & $x$ & & & & & & & \\
\hline \multirow{2}{*}{ Complications } & & & & & & & & \\
\hline & & & $x$ & & & & & \\
\hline Hospital Stay & $x$ & & & & & $\rightarrow$ & & \\
\hline \multicolumn{9}{|l|}{ Analgesics } \\
\hline $\begin{array}{r}\text { Radiographs } \\
\text { (standing) }\end{array}$ & & & $X$ & & $x$ & $x$ & $x$ & $x$ \\
\hline \multirow{2}{*}{$E Q-5 D$} & & & $x$ & & & $X$ & $x$ & $\mathrm{x}$ \\
\hline & \multicolumn{8}{|c|}{$O D I$} \\
\hline & & & & & & & & \\
\hline \multicolumn{9}{|l|}{ Return to work } \\
\hline $\begin{array}{l}\text { Subjective } \\
\text { outcomes }\end{array}$ & & & & & & $x$ & & \\
\hline $\begin{array}{r}\text { Orthosis } \\
\text { compliance }\end{array}$ & & & & & & & & \\
\hline
\end{tabular}

Figure 2 Flow diagram of enrolment, intervention and outcome assessment according to SPIRIT guidelines. EQ-5D, EuroQuol 5 Dimensions; NRS, Numerical Rating Scale; ODI, Oswestry Disability Index; SPIRIT, Standard Protocol Items: Recommendations for Interventional Trials.

\section{Complications}

All complications occurring during the study period from inclusion to final follow-up after 1 year are registered. Complications are extracted from the hospital information system. Complications will include, but will not be limited to, infections (pulmonary, urinary tract and wound) that are culture proven and/or treated with antibiotics, clinically diagnosed and registered pressure sores, ileus treated with a gastric tube and deep venous thrombosis that is ultrasound proven and medically treated.
Complications will be graded according to the ClavienDindo grading scale. ${ }^{37}$

\section{Hospital stay and return to work}

Hospital stay is defined as the amount of days from surgery to discharge. Return to work is defined as the amount of days from surgery to the outpatient appointment on which it is first registered that a patient has returned to work. Results are stratified for patients that have partially returned to work. 


\section{Subjective outcomes}

After 12 weeks, the group that was randomised to using an orthosis will receive three additional subjective questions accompanying the validated questionnaires. These consist of orthosis satisfaction (yes/no), wishing a postoperative orthosis if another thoracolumbar fracture would occur (yes/no), main benefits of the orthosis (pain relief, confidence, faster healing, reminder to take care, did not help, other (open)).

\section{Orthosis compliance}

Additionally, the group that was randomised to an orthosis will receive a questionnaire at inclusion on which they are asked to register orthosis compliance. Compliance should be registered weekly, at the end of every week, for 12 weeks. An estimate has to be given of how often the orthosis was worn as it was required by the physiotherapist or doctor. Answers consist of always $(100 \%)$, most of the time $(75 \%)$, half of the time $(50 \%)$, quarter of the time $(25 \%)$ or never $(0 \%)$. On every measurement moment of other questionnaires (2, 6 and 12 weeks), patients are also reminded to fill in the pain and compliance forms.

\section{Data collection}

All data will be collected, coded and stored using Castor EDC (www.castoredc.com), a Good Clinical Practice guideline approved online data-capture application. The decoding file will be saved to a local secured hospital drive, only accessible by the principal and coordinating researchers. Data are collected during hospital stay, at specific measurement moments and outpatient appointments (figure 2). Questionnaires will be sent by email through Castor. The coordinating investigator (AJS) regularly checks if questionnaires are sent and completed, if not, the questionnaire is generally resent within 5 days. If the questionnaire is not completed within 1 week, the patient is called by the coordinating investigator. Baseline data and secondary outcomes including NRS during hospital stay (standard nursing care) will be extracted from the hospital information system. No additional outpatient appointments will be made for study purposes and radiographic investigations will only be ordered on behalf of necessary treatment by the treating surgeon, although this generally follows a standard schedule. A study flow diagram is shown in figure 1 and detailed follow-up in figure 2. Patients who deviate from intervention are requested to report this on the orthosis compliance form with arguments and to continue reporting outcomes as they would have otherwise.

\section{Intervention assignment}

Intervention allocation is computer generated using Castor, randomisation is stratified for single or multiple vertebral fractures and treatment centre. These variables are used for stratification of randomisation because these possibly influence the primary outcome (pain). As this study is not designed to change local daily practice of surgical techniques concerning the approach (minimally invasive surgery (MIS) vs open surgery) or the use of additional transpedicular vertebral body stenting (VBS), patients are stratified for treatment centre. Choice of surgical technique is based on patient and fracture characteristics and surgeons preferences in both hospitals. One hospital preferably treats patients with minimally invasive techniques and is liberal in the use of VBS (Onze Lieve Vrouwe Gasthuis). The other hospital uses both open and minimally invasive techniques, and is less liberal in the use of VBS (VU University Medical Center). Patients can only be randomised by the principal researcher and the coordinating researcher, and after informed consent is signed. Allocation is not blinded to patients, researchers and care providers as this is practically not possible. The outcome assessor of kyphosis and fracture consolidation will be blinded to patient allocation.

\section{Sample size}

A power calculation based on the primary outcome parameter was done to calculate the required sample size. The primary outcome is pain on NRS score at 6 weeks postoperative. There is currently no literature available that describes the minimal clinically important difference (MCID) for acute traumatic spine fractures. It has however been stated by experts that there is insufficient empirical evidence to set different minimal important change (MIC) values for acute or chronic low back pain. ${ }^{22}$ Based on the expertise of the initiating hospital's pain consulting team (that treats a lot of patients with acute spine fractures) and existing literature, ${ }^{21} 22$ a difference of 2 points on NRS score with SD of 2.5 was used as a clinically significant difference. Using these differences, a significance level of $\alpha=0.05$ and power of $\beta=0.80$, it was calculated that with 21 patients in both groups a power of $\beta=0.82$ can be reached. When taking a follow-up loss of $10 \%$ into account, the total amount of patients aimed for is 46 .

\section{Statistics}

Outcomes will be analysed using IBM SPSS V.22. Primary analysis will be done according to both intention-to-treat principle and per-protocol approach, due to the non-inferiority set-up. ${ }^{38-40}$ Non-compliant patients will not be crossed over the other group as this would probably lead to an overestimation of the no-orthosis group. Depending on the amount of non-compliance of the orthosis, it will be decided which analysis is of more importance if they do not lead to the same conclusion.

Categorical data will be displayed as frequencies with proportions. Continuous outcomes will be described as mean with SD and if skewed as median with IQR. Normality will be tested visually using a histogram, $\mathrm{Q}-\mathrm{Q}$ plot and box plot. Parametric tests will be used if data follow a normal distribution and non-parametric tests otherwise. The primary outcome, average NRS score on 6 weeks, will be analysed using an independent t-test or Mann-Whitney U test if skewed. 
To compare secondary outcomes at specific time moments, depending on normality, a t-test or MannWhitney $\mathrm{U}$ test will be used. In case of confounding or effect modification, this will be corrected for using linear regression analysis. To analyse the effect on NRS, ODI and EQ-5D over time, a mixed model analysis for repeated measures will be performed. Categorical variables will be analysed using a $\chi^{2}$ test.

\section{Monitoring}

Data monitoring is provided by an independent clinical research bureau (Clinical Research Bureau, Amsterdam, the Netherlands) and will take place at predefined moments in the initiating centre based on study risk classification. Monitoring in the initiating centre is deemed sufficient as the study risk has been classified negligible and most inclusions are expected in the initiating centre. Monitoring starts after approval of the study by the research ethics committee/institutional review board (REC/IRB). Principal researchers will be informed orally and in writing on the results of monitoring. The REC/ IRB will be informed annually with a progress report. All (serious) adverse events and serious adverse device events will be reported in due time to, and judged by, the REC/ IRB.

\section{ETHICS AND DISSEMINATION}

There has so far been one approved protocol modification consisting of an added centre, transforming the study from single centre to multicentre. All protocol modifications will only be done after approval of the REC/IRB. An interim analysis is done after inclusion of the first 20 patients. If a statistical difference of $\geq 3$ NRS points between groups is found, the METc will be consulted to judge if premature termination is necessary. The study has been registered in an international and a national trial database, respectively ClinicalTrials.gov (NCT03097081) and trialregister.nl (NTR6285. After completion of the trial, the results will be offered to an international scientific journal for peer-reviewed publication.

\section{DISCUSSION}

The role of an orthosis in postoperative care of surgically treated thoracolumbar fractures has not yet been studied properly. An essential role of the orthosis in the healing process of these injuries has never been proven, which explains the current subjective use in postoperative care. ${ }^{23}$ The beneficial effect can only be scientifically tested by randomising groups between the use and no use of an orthosis. To our best knowledge, this is the first prospective randomised study on the use of postoperative orthoses.

Patients randomised to an orthosis might not use it as prescribed. Therefore, weekly monitoring of orthosis compliance is introduced in this study. Some dependence on patients' willingness to truthfully and periodically complete the questionnaires (pain and orthosis compliance) is consequently created. However, despite reminders some missing data cannot be ruled out. A compliance validation study is however currently being planned using temperature sensors to obtain true orthosis compliance data. If this study will start while the current study is still including patients, some will receive an orthosis with a temperature sensor. The validation of the compliance forms will then be reported in the study results and used for final analysis.

While other studies on orthoses for spine fractures usually measure pain only once for each moment, ${ }^{11} 1641$ pain measurement in this study consists of several consecutive scores. Even with momentary peaks or missing values, a very reliable average outcome can be computed.

One centre was added 6 months after the first inclusion, changing the study from monocentre to multicentre. Introducing some heterogeneity as local operative treatment protocols differ slightly between hospitals due to different standards of surgical practice. This concerns the posterior open versus minimally invasive approach and the additional use of VBS. ${ }^{42}$ As randomisation is stratified for participating centre, it is unlikely that this outcome will be influenced unequally. Furthermore, the number of patients treated with MIS and VBS in each group will be reported and outcomes will be statistically corrected if needed. Additionally, it is likely that these techniques will in the future become standard care, making the results of this study wider applicable. No patients with a psychiatric comorbidity have been included so far, and this could considerably influence QOL outcomes. Therefore, an additional amendment that excludes patients with a psychiatric comorbidity to participate in the study is currently under consideration by the IRB.

\section{CONCLUSION}

This prospective randomised non-inferiority study will provide a clear recommendation on the use of a postoperative orthosis after posteriorly stabilised thoracolumbar fractures. If no orthosis is proven to be non-inferior to an orthosis, this could lead to a change in postoperative protocols in which orthoses should not be used routinely. This could result in less unnecessary patient inconvenience and less costs.

\section{TRIAL STATUS}

The trial has started recruiting patients on 29 November 2016 and is currently under protocol V.4 designed at 8 August 2017. Recruitment is expected to be complete by the end of 2018 .

Contributors AJS, JD and FWB designed the trial and are principal investigators. AJS reviewed literature, acquired data and drafted the manuscript. JD, FWB, MCA, DHRK and AS contributed to the drafting of the manuscript. All authors read and approved the final manuscript.

Funding This research received no specific grant from any funding agency in the public, commercial or not-for-profit sectors. 
Competing interests None declared.

Ethics approval The study protocol has first been approved by the MOVE research institute affiliated with VUmc Amsterdam on 18 July 2016 (protocol number 16.06). The ResearchEthics Committee/Institutional Review Board (METc VUmc) approved the study on 11 October 2016 (case number 2016.389).

Provenance and peer review Not commissioned; externally peer reviewed.

Open Access This is an Open Access article distributed in accordance with the Creative Commons Attribution Non Commercial (CC BY-NC 4.0) license, which permits others to distribute, remix, adapt, build upon this work non-commercially, and license their derivative works on different terms, provided the original work is properly cited and the use is non-commercial. See: http://creativecommons.org/ licenses/by-nc/4.0/

(C) Article author(s) (or their employer(s) unless otherwise stated in the text of the article) 2018. All rights reserved. No commercial use is permitted unless otherwise expressly granted.

\section{REFERENCES}

1. Joaquim AF, Patel AA. Thoracolumbar spine trauma: Evaluation and surgical decision-making. J Craniovertebr Junction Spine 2013;4:3-9.

2. Kepler CK, Vroome C, Goldfarb M, et al. Variation in the management of thoracolumbar trauma and postoperative infection. J Spinal Disord Tech 2015;28:E212-8.

3. Skoch J, Zoccali C, Zaninovich O, et al. Bracing after surgical stabilization of thoracolumbar fractures: a systematic review of evidence, indications, and practices. World Neurosurg 2016:93:221-8.

4. Rohlmann A, Zander T, Graichen F, et al. Effect of an orthosis on the loads acting on a vertebral body replacement. Clin Biomech 2013;28:490-4

5. Aono $\mathrm{H}$, Tobimatsu $\mathrm{H}$, Ariga $\mathrm{K}$, et al. Surgical outcomes of temporary short-segment instrumentation without augmentation for thoracolumbar burst fractures. Injury 2016;47:1337-44.

6. Parker JW, Lane JR, Karaikovic EE, et al. Successful short-segment instrumentation and fusion for thoracolumbar spine fractures: a consecutive 41/2-year series. Spine 2000;25:1157-70.

7. Shen WJ, Liu TJ, Shen YS. Nonoperative treatment versus posterior fixation for thoracolumbar junction burst fractures without neurologic deficit. Spine 2001;26:1038-45.

8. Jiang XZ, Tian W, Liu B, et al. Comparison of a paraspinal approach with a percutaneous approach in the treatment of thoracolumbar burst fractures with posterior ligamentous complex injury: a prospective randomized controlled trial. J Int Med Res 2012:40:1343-56.

9. Jindal N, Sankhala SS, Bachhal V. The role of fusion in the management of burst fractures of the thoracolumbar spine treated by short segment pedicle screw fixation: a prospective randomised trial. J Bone Joint Surg Br 2012;94:1101-6.

10. Bailey CS, Dvorak MF, Thomas KC, et al. Comparison of thoracolumbosacral orthosis and no orthosis for the treatment of thoracolumbar burst fractures: interim analysis of a multicenter randomized clinical equivalence trial. J Neurosurg Spine 2009;11:295-303.

11. Kim HJ, Yi JM, Cho HG, et al. Comparative study of the treatment outcomes of osteoporotic compression fractures without neurologic injury using a rigid brace, a soft brace, and no brace: a prospective randomized controlled non-inferiority trial. J Bone Joint Surg Am 2014;96:1959-66.

12. Gelb D, Ludwig S, Karp JE, et al. Successful treatment of thoracolumbar fractures with short-segment pedicle instrumentation. J Spinal Disord Tech 2010;23:293-301.

13. Moelmer M, Gehrchen M, Dahl B. Long-term functional results after short-segment pedicle fixation of thoracolumbar fractures. Injury 2013;44:1843-6.

14. Connolly PJ, Grob D. Bracing of patients after fusion for degenerative problems of the lumbar spine--yes or no? Spine 1998;23:1426-8.

15. Yee AJ, Yoo JU, Marsolais EB, et al. Use of a postoperative lumbar corset after lumbar spinal arthrodesis for degenerative conditions of the spine. A prospective randomized trial. J Bone Joint Surg Am 2008;90:2062-8.

16. Bailey CS, Urquhart JC, Dvorak MF, et al. Orthosis versus no orthosis for the treatment of thoracolumbar burst fractures without neurologic injury: a multicenter prospective randomized equivalence trial. Spine J 2014;14:2557-64.

17. Shamji MF, Roffey DM, Young DK, et al. A pilot evaluation of the role of bracing in stable thoracolumbar burst fractures without neurological deficit. J Spinal Disord Tech 2014;27:370-5.

18. Stadhouder A, Buskens E, Vergroesen DA, et al. Nonoperative treatment of thoracic and lumbar spine fractures: a prospective randomized study of different treatment options. J Orthop Trauma 2009;23:588-94.

19. Chan AW, Tetzlaff JM, Gøtzsche PC, et al. SPIRIT 2013 explanation and elaboration: guidance for protocols of clinical trials. BMJ 2013;346:e7586.

20. Moher D, Hopewell S, Schulz KF, et al. CONSORT 2010 explanation and elaboration: updated guidelines for reporting parallel group randomised trials. Int J Surg 2012;10:28-55.

21. Jensen MP. Pain assessment in clinical trials. In: Wittink HM, Carr DB, eds. Pain management: evidence, outcomes, and quality of life a sourcebook. Amsterdam, The Netherlands: Elsevier, 2008:57-81.

22. Ostelo RW, Deyo RA, Stratford P, et al. Interpreting change scores for pain and functional status in low back pain: towards international consensus regarding minimal important change. Spine 2008;33:90-4.

23. Bijur PE, Latimer CT, Gallagher EJ. Validation of a verbally administered numerical rating scale of acute pain for use in the emergency department. Acad Emerg Med 2003;10:390-2.

24. Ferreira-Valente MA, Pais-Ribeiro JL, Jensen MP. Validity of four pain intensity rating scales. Pain 2011;152:2399-404.

25. Price DD, Bush FM, Long S, et al. A comparison of pain measurement characteristics of mechanical visual analogue and simple numerical rating scales. Pain 1994;56:217-26.

26. de C Williams AC, Davies HT, Chadury Y. Simple pain rating scales hide complex idiosyncratic meanings. Pain 2000;85:457-63.

27. Chapman JR, Norvell DC, Hermsmeyer JT, et al. Evaluating common outcomes for measuring treatment success for chronic low back pain. Spine 2011;36:S54-68.

28. Childs JD, Piva SR, Fritz JM. Responsiveness of the numeric pain rating scale in patients with low back pain. Spine 2005;30:1331-4.

29. Pengel LH, Refshauge KM, Maher CG. Responsiveness of pain, disability, and physical impairment outcomes in patients with low back pain. Spine 2004;29:879-83.

30. Jensen MP, McFarland CA. Increasing the reliability and validity of pain intensity measurement in chronic pain patients. Pain 1993;55:195-203.

31. Soer R, Reneman MF, Speijer BL, et al. Clinimetric properties of the EuroQol-5D in patients with chronic low back pain. Spine $J$ 2012;12:1035-9.

32. Agota Szende BJ, Cabases J. Self-reported population health: an international perspective based on EQ-5D. The Netherlands: Springer, 2014:196.

33. Fairbank JC, Couper J, Davies JB, et al. The Oswestry low back pain disability questionnaire. Physiotherapy 1980;66:271-3.

34. Roland M, Fairbank J. The Roland-Morris disability questionnaire and the oswestry disability questionnaire. Spine 2000;25:3115-24.

35. Scheer JK, Bakhsheshian J, Fakurnejad S, et al. Evidence-based medicine of traumatic thoracolumbar burst fractures: a systematic review of operative management across 20 years. Global Spine $J$ 2015;5:73-82.

36. Kuklo TR, Polly DW, Owens BD, et al. Measurement of thoracic and lumbar fracture kyphosis: evaluation of intraobserver, interobserver, and technique variability. Spine 2001;26:61-5.

37. Dindo D, Demartines N, Clavien PA. Classification of surgical complications: a new proposal with evaluation in a cohort of 6336 patients and results of a survey. Ann Surg 2004:240:205-13.

38. Le Henanff A, Giraudeau B, Baron G, et al. Quality of reporting of noninferiority and equivalence randomized trials. JAMA 2006;295:1147-51.

39. Wiens BL, Zhao W. The role of intention to treat in analysis of noninferiority studies. Clin Trials 2007;4:286-91.

40. Piaggio G, Elbourne DR, Altman DG, et al. Reporting of noninferiority and equivalence randomized trials: an extension of the CONSORT statement. JAMA 2006;295:1152-60.

41. Wood K, Buttermann G, Butterman G, et al. Operative compared with nonoperative treatment of a thoracolumbar burst fracture without neurological deficit. A prospective, randomized study. J Bone Joint Surg Am 2003;85-A:773-81.

42. Rotter R, Martin H, Fuerderer S, et al. Vertebral body stenting: a new method for vertebral augmentation versus kyphoplasty. Eur Spine J 2010;19:916-23. 